\title{
Use and effectiveness of pegfilgrastim prophylaxis in US clinical practice:a retrospective observational study
}

Derek Weycker ${ }^{1 *} \mathbb{D}$, Robin Doroff ${ }^{1}$, Ahuva Hanau', Charles Bowers ${ }^{2}$, Rajesh Belani $^{2}$, David Chandler², Alexander Lonshteyn ${ }^{1}$, Mark Bensink² and Gary H. Lyman ${ }^{3}$

\begin{abstract}
Background: Febrile neutropenia (FN) is a serious complication of myelosuppressive chemotherapy. Clinical practice guidelines recommend routine prophylactic coverage with granulocyte colony-stimulating factor (G-CSF)—such as pegfilgrastim—for most patients receiving chemotherapy with an intermediate to high risk for FN. Patterns of pegfilgrastim prophylaxis during the chemotherapy course and associated FN risks in US clinical practice have not been well characterized.

Methods: A retrospective cohort design and data from two commercial healthcare claims repositories (01/2010-03/ 2016) and Medicare Claims Research Identifiable Files (01/2007-09/2015) were employed. Study population included patients who had non-metastatic breast cancer or non-Hodgkin's lymphoma and received intermediate/high-risk regimens. Pegfilgrastim prophylaxis use and FN incidence were ascertained in each chemotherapy cycle, and all cycles were pooled for analyses. Adjusted odds ratios for FN were estimated for patients who did versus did not receive pegfilgrastim prophylaxis in that cycle.

Results: Study population included 50,778 commercial patients who received 190,622 cycles of chemotherapy and 71, 037 Medicare patients who received 271,944 cycles. In cycle 1, 33\% of commercial patients and 28\% of Medicare patients did not receive pegfilgrastim prophylaxis, and adjusted odds of FN were $2.6(95 \% \mathrm{Cl} 2.3-2.8)$ and $1.6(1.5-1.7)$, respectively, versus those who received pegfilgrastim prophylaxis. In cycle 2, 28\% (commercial) and 26\% (Medicare) did not receive pegfilgrastim prophylaxis; corresponding adjusted FN odds were comparably elevated (1.9 [1.6-2.2] and 1.6 [1.5-1.8]). Results in subsequent cycles were similar. Across all cycles, $15 \%$ of commercial patients and 23\% of Medicare patients did not receive pegfilgrastim prophylaxis despite having FN in a prior cycle, and prior FN increased odds of subsequent FN by 2.1-2.4 times.

Conclusions: Notwithstanding clinical practice guidelines, a large minority of patients did not receive G-CSF prophylaxis, and FN incidence was substantially higher among this subset of the population. Appropriate use of pegfilgrastim prophylaxis may reduce patient exposure to this potentially fatal but largely preventable complication of myelosuppressive chemotherapy.
\end{abstract}

Keywords: Febrile neutropenia, Pegfilgrastim, Neulasta, Granulocyte colony-stimulating factor

\footnotetext{
* Correspondence: dweycker@pai2.com

${ }^{1}$ Policy Analysis Inc. (PAI), Four Davis Court, Brookline, MA 02445, USA

Full list of author information is available at the end of the article
}

(c) The Author(s). 2019 Open Access This article is distributed under the terms of the Creative Commons Attribution 4.0 International License (http://creativecommons.org/licenses/by/4.0/), which permits unrestricted use, distribution, and reproduction in any medium, provided you give appropriate credit to the original author(s) and the source, provide a link to the Creative Commons license, and indicate if changes were made. The Creative Commons Public Domain Dedication waiver (http://creativecommons.org/publicdomain/zero/1.0/) applies to the data made available in this article, unless otherwise stated. 


\section{Background}

Low neutrophil count ("neutropenia") is a frequent side effect of myelotoxic chemotherapy and increases infection risk. Neutropenia in the presence of fever ("febrile neutropenia" [FN]) typically necessitates inpatient care and may result in delays, reductions, and/or discontinuation of chemotherapy that can-in turn-lead to adverse outcomes [1-11]. For patients whose projected FN risk is high ( $>20 \%$ ) based on the planned chemotherapy regimen and individual risk factors (e.g., age $>65$ years, comorbidity profile), prophylaxis with granulocyte colony-stimulating factor (G-CSF) is recommended $[1,9$, 12-14]. However, published evidence indicates that many patients who are candidates for G-CSF are not administered it, or are not administered it per recommendations, and thus may be at elevated risk of FN and hospitalization [12-27].

Pegfilgrastim, which requires only a single dose per cycle of chemotherapy, is the most commonly used CSF agent in the US, with previous evaluations reporting that it accounted for $>90 \%$ of all CSF prophylaxis use in their study populations [12, 15, 20, 24, 28, 29]. Moreover, pegfilgrastim has been reported-in post-hoc analyses of clinical trials, meta-analyses of clinical trials, and realworld evaluations - to be more efficacious and effective than other CSFs in preventing FN [12, 15, 20, 28-33]. Notwithstanding the availability of pegfilgrastim since 2002 and clinical practice guidelines supporting its use, relatively little is known about patterns of pegfilgrastim use across multiple cycles of chemotherapy during the course, the influence of FN on subsequent pegfilgrastim use, and the impact of pegfilgrastim on the incidence of FN [34]. We therefore undertook two retrospective observational cohort studies, the first using data from two large healthcare claims repositories and the second using data from Medicare Claims Research Identifiable Files (RIFs), to examine these issues among patients with non-metastatic breast cancer or non-Hodgkin's lymphoma (NHL) receiving chemotherapy regimens with an intermediate to high risk for FN in US clinical practice.

\section{Methods}

The methods of this study-including the design, identification of source/study populations, and variable definitionsare largely the same as those employed in prior evaluations conducted by some of the investigators involved in the present research [16, 17, 23, 35]. An additional file provides a detailed description of study methods and source databases (Additional file 1); a brief description follows.

\section{Study design and data source}

A retrospective observational cohort design was employed to analyze patient-level data from commercial claims and Medicare claims, respectively. For commercial claims
(January 2010 - March 2016), data were obtained from two repositories: the Truven Health Analytics Market$\mathrm{Scan}^{\bullet}$ Commercial Claims and Encounters and Medicare Supplemental and Coordination of Benefits Databases (MarketScan Database) and the IMS LifeLink ${ }^{\mathrm{Tm}}$ PharMetrics Plus Health Plan Claims Database (PharMetrics Plus Database). For Medicare claims (January 2007 - September 2015), data were obtained from the RIFs of the Centers for Medicare and Medicaid Services (CMS). Data extracts were de-identified prior to their release to study investigators and thus their use for health services research is compliant with the Health Insurance Portability and Accountability Act (HIPAA) Privacy Rule and federal guidance on Public Welfare and the Protection of Human Subjects, and Institutional Review Board (IRB) status is exempt [36].

\section{Source and study populations}

For commercial patients, the source population included all patients aged $\geq 18$ years who, from July 1, 2010 through September 30, 2015, received myelosuppressive chemotherapy for solid tumors or NHL. For Medicare patients, the source population included all patients aged $\geq 65$ years who, from July 1, 2007 through March 31, 2015, received myelosuppressive chemotherapy for solid tumors or NHL. In both the commercial and Medicare source populations, patients with $<6$ months of continuous health benefits prior to initiation of chemotherapy, evidence of multiple primary cancers, or who did not meet other inclusion/exclusion criteria (as described in Additional file 1) were excluded.

From the source populations, all patients with non-metastatic breast cancer or NHL who received selected chemotherapy regimens with an intermediate/high-risk for FN were included in the study population. Selected intermediate/high-risk regimens included those commonly used in US clinical practice: docetaxel + doxorubicin + cyclophosphamide (TAC), docetaxel + cyclophosphamide (TC), and docetaxel + carboplatin + trastuzumab $(\mathrm{TCH})$ for nonmetastatic breast cancer; and cyclophosphamide + doxorubicin + vincristine + prednisone \pm rituximab $(\mathrm{CHOP} \pm \mathrm{R})$ for NHL.

For each patient in the study population, each cycle of chemotherapy within the first qualifying course was characterized and use of pegfilgrastim prophylaxis on days 1-3 from the last administration of chemotherapy was ascertained in each cycle. Chemotherapy courses were limited to the first 8 cycles and were truncated if there was an unplanned switch in regimen (i.e., an unplanned change in agents administered in subsequent cycles versus the first cycle).

All patient-cycles meeting the following additional criteria were pooled for analyses: no prophylaxis with other CSF agents (i.e., use of filgrastim, tbo-filgrastim, sargramostim on the same day as chemotherapy or days $1-5$ 
following last receipt of chemotherapy); no prophylaxis with antimicrobials; no receipt of pegfilgrastim on the same day as chemotherapy or days 4-5 following last receipt of chemotherapy; and no evidence of FN prior to administration of pegfilgrastim in that cycle.

\section{Febrile neutropenia}

FN episodes were ascertained in each chemotherapy cycle, from the fourth day following the last receipt of chemotherapy through the last day of the cycle, using a "broad" algorithm and a "narrow" algorithm [15-17, 19, 21, 23, 24, 28, 35, 37]. For the broad algorithm, FN was ascertained in the inpatient setting based on a diagnosis (principal or secondary) of neutropenia, fever, or infection, and in the outpatient setting based on a diagnosis of neutropenia, fever, or infection and-on the same date-IV administration of antimicrobial therapy. For the narrow algorithm, FN was ascertained in the inpatient setting based on a diagnosis (principal or secondary) of neutropenia, and in the outpatient setting based on a diagnosis of neutropenia and-on the same date-administration of IV antimicrobial therapy.

\section{Statistical analyses}

Unadjusted incidence proportions for use of pegfilgrastim prophylaxis (overall and by FN occurrence [broad algorithm] in a prior cycle) and incidence proportions for FN based on the broad algorithm (overall and for subgroups defined on receipt of pegfilgrastim prophylaxis in that cycle) were summarized on a cycle-specific basis. Corresponding unadjusted odds ratios (ORs) were estimated using generalized estimating equations (GEEs), as described below.

ORs for $\mathrm{FN}$ in a given cycle were also estimated for patients who did versus did not receive pegfilgrastim in that cycle, with adjustment for FN in a previous cycle, chemotherapy regimen, age, and other covariates (as described in Additional file 1) using GEEs with a binomial distribution, logistic link function, and exchangeable correlation structure. The GEE method accounts for correlation among repeated measures for the same patient (in this instance, among cycles), while controlling for both variables that are invariant as well as those that may vary across observations. These analyses were conducted for all patients in the study population using the broad and narrow algorithms for FN in cycle 1 , cycle 2 , cycles $\geq 3$, the last cycle, and all cycles. All analyses were conducted at the level of the chemotherapy cycle, and analyses were conducted using commercial claims and Medicare claims separately. Other covariates were selected for inclusion in regression models via a backward selection method $(p<0.10)$, and included patient, cancer, and treatment characteristics listed in Additional file 1.

\section{Results}

Patient characteristics

A total of 50,778 commercial patients with non-metastatic breast cancer or NHL received 190,622 cycles of intermediate/high-risk chemotherapy during the study period and met all other criteria for inclusion. Among Medicare patients with non-metastatic breast cancer or NHL, 71,037 received 271,944 cycles of intermediate/ high-risk chemotherapy during the study period and met all other criteria for inclusion. Patient characteristics were generally comparable between those who received pegfilgrastim in cycle 1 and those who did not; although some characteristics were statistically different between subgroups, the observed variation in values was not clinically meaningful (Table 1).

\section{Patterns of Pegfilgrastim prophylaxis and crude FN risk}

In cycle $1,67 \%$ of commercial patients and $72 \%$ of Medicare patients received pegfilgrastim prophylaxis (Table 2). Among commercial patients, use of pegfilgrastim prophylaxis was comparable in cycle 2 (72\%), subsequent cycles (75\%), and the last cycle (71\%). Among Medicare patients, use of pegfilgrastim prophylaxis in cycle 2 (74\%) and subsequent cycles $(70 \%)$ was comparable. In the last chemotherapy cycle, however, overall pegfilgrastim use was lower (61\%). Across all cycles, use of pegfilgrastim prophylaxis was somewhat higher among patients who had FN in a prior cycle (85\% for commercial patients and $77 \%$ for Medicare patients).

The unadjusted FN incidence among commercial and Medicare patients was 3.0 and $6.2 \%$ across all cycles, 5.2 and $9.6 \%$ in cycle $1,2.3$ and $5.2 \%$ in cycle 2 , and 2.1 and $5.0 \%$ in subsequent cycles, respectively (Table 3). For both commercial and Medicare patients, FN incidence was generally lower among patients who received pegfilgrastim prophylaxis versus those who did not.

\section{Multivariable analysis of FN}

After adjustment for differences in age, chemotherapy regimen, and other covariates, odds of FN over all cycles (broad algorithm) were 2.1 times higher (95\% CI 2.0-2.3) among commercial patients and 1.5 times higher (95\% CI 1.4-1.5) among Medicare patients who did not receive pegfilgrastim prophylaxis versus those who did (Table 4). In cycle 1, FN odds were 2.6 times higher (95\% CI 2.3-2.8) among commercial patients and 1.6 times higher (95\% CI 1.5-1.7) among Medicare patients. After adjustment for the aforementioned factors as well as FN in a prior cycle, FN odds in cycle 2 and subsequent cycles were similarly elevated for those not receiving prophylaxis among both commercial and Medicare patients.

For commercial patients, odds of FN across all cycles were substantially higher among those who had an FN event in a prior cycle $(\mathrm{OR}=2.4 ; 95 \%$ CI 2.2-2.6), were 
Table 1 Characteristics of study population, by use of pegfilgrasim prophylaxis in cycle 1

\begin{tabular}{|c|c|c|c|c|}
\hline & \multicolumn{2}{|l|}{ Commercial } & \multicolumn{2}{|l|}{ Medicare } \\
\hline & $\begin{array}{l}\text { No Pegfilgrastim Use } \\
(n=16,512)\end{array}$ & $\begin{array}{l}\text { Pegfilgrastim Use } \\
(n=34,266)\end{array}$ & $\begin{array}{l}\text { No Pegfilgrastim Use } \\
(n=20,195)\end{array}$ & $\begin{array}{l}\text { Pegfilgrastim Use } \\
(n=50,842)\end{array}$ \\
\hline \multicolumn{5}{|l|}{ Patient } \\
\hline \multicolumn{5}{|l|}{ Age (years) } \\
\hline Mean (SD) & $54.3(10.2)$ & $56.2(10.9)$ & $72.3(5.4)$ & $73.6(5.9)$ \\
\hline Female, N (\%) & $14,623(89 \%)$ & $29,995(88 \%)$ & $16,604(82 \%)$ & $37,811(74 \%)$ \\
\hline \multicolumn{5}{|l|}{ Chronic Comorbidities, N (\%) } \\
\hline Cardiovascular Disease & $1403(8 \%)$ & $3851(11 \%)$ & $6124(30 \%)$ & $16,979(33 \%)$ \\
\hline Diabetes & 1750 (11\%) & $4211(12 \%)$ & $5217(26 \%)$ & $13,022(26 \%)$ \\
\hline Liver Disease & $621(4 \%)$ & $1386(4 \%)$ & $731(4 \%)$ & $1999(4 \%)$ \\
\hline Lung Disease & $1290(8 \%)$ & $3157(9 \%)$ & $3033(15 \%)$ & $8053(16 \%)$ \\
\hline Renal Disease & $311(2 \%)$ & $855(2 \%)$ & $1523(8 \%)$ & $4598(9 \%)$ \\
\hline Osteoarthritis & $1382(8 \%)$ & $3361(10 \%)$ & $4534(22 \%)$ & $11,620(23 \%)$ \\
\hline Rheumatoid Disease & $236(1 \%)$ & $561(2 \%)$ & $670(3 \%)$ & $1798(4 \%)$ \\
\hline Thyroid Disorder & $2105(13 \%)$ & 4569 (13\%) & $4470(22 \%)$ & $11,723(23 \%)$ \\
\hline \multicolumn{5}{|l|}{ Body Weight and Nutritional Status, N (\%) } \\
\hline Obese & $1244(8 \%)$ & $2688(8 \%)$ & $1715(8 \%)$ & $3959(8 \%)$ \\
\hline Malnutrition & $71(0 \%)$ & $289(1 \%)$ & $414(2 \%)$ & $1517(3 \%)$ \\
\hline \multicolumn{5}{|l|}{ Proxies for Health Status, N (\%) } \\
\hline Hospice Care & $38(0 \%)$ & $120(0 \%)$ & $572(3 \%)$ & $2037(4 \%)$ \\
\hline SNF & $100(1 \%)$ & $244(1 \%)$ & $926(5 \%)$ & $2194(4 \%)$ \\
\hline Hospice or SNF & $136(1 \%)$ & $352(1 \%)$ & $1400(7 \%)$ & $3962(8 \%)$ \\
\hline \multicolumn{5}{|l|}{ Proxies for Physical Function, N (\%) } \\
\hline Use of Hospital Bed & $26(0 \%)$ & $78(0 \%)$ & $162(1 \%)$ & $476(1 \%)$ \\
\hline Use of Supplemental Oxygen & $259(2 \%)$ & $681(2 \%)$ & $810(4 \%)$ & $2285(4 \%)$ \\
\hline Use of Walking Aid & $192(1 \%)$ & $506(1 \%)$ & $772(4 \%)$ & $2455(5 \%)$ \\
\hline Use of Wheel Chair & $53(0 \%)$ & $136(0 \%)$ & $378(2 \%)$ & $997(2 \%)$ \\
\hline Any of Above & $487(3 \%)$ & $1256(4 \%)$ & $1807(9 \%)$ & $5268(10 \%)$ \\
\hline Use of Immunosuppressive Drugs, N (\%) & $5273(32 \%)$ & $11,113(32 \%)$ & $6612(33 \%)$ & $17,145(34 \%)$ \\
\hline \multicolumn{5}{|c|}{ Other Conditions/Events Prior to Chemotherapy, N (\%) } \\
\hline Anemia & $1896(11 \%)$ & $4721(14 \%)$ & $6025(30 \%)$ & $17,970(35 \%)$ \\
\hline Neutropenia & $290(2 \%)$ & $1295(4 \%)$ & $948(5 \%)$ & $4738(9 \%)$ \\
\hline Infection & $7902(48 \%)$ & $17,204(50 \%)$ & $11,545(57 \%)$ & $29,868(59 \%)$ \\
\hline Recent Surgery (prior 90 days) & $13,881(84 \%)$ & $28,100(82 \%)$ & $13,024(64 \%)$ & $30,427(60 \%)$ \\
\hline History of Hospitalization for Any Reason & $4549(28 \%)$ & 9985 (29\%) & 7739 (38\%) & $20,374(40 \%)$ \\
\hline History of Chemotherapy & $866(5 \%)$ & $2127(6 \%)$ & $1623(8 \%)$ & $4755(9 \%)$ \\
\hline History of Radiation Therapy & $910(6 \%)$ & $1955(6 \%)$ & $883(4 \%)$ & $2140(4 \%)$ \\
\hline \multicolumn{5}{|l|}{ Cancer and Chemotherapy, N (\%) } \\
\hline Breast Cancer & $13,261(80 \%)$ & $26,622(78 \%)$ & $13,305(66 \%)$ & $25,675(50 \%)$ \\
\hline TAC & $389(2 \%)$ & 4179 (12\%) & $235(1 \%)$ & $1857(4 \%)$ \\
\hline TC & $8793(53 \%)$ & $14,918(44 \%)$ & $8032(40 \%)$ & $19,493(38 \%)$ \\
\hline $\mathrm{TCH}$ & $4079(25 \%)$ & $7525(22 \%)$ & $5038(25 \%)$ & $4325(9 \%)$ \\
\hline $\mathrm{NHL}-\mathrm{CHOP} \pm \mathrm{R}$ & $3251(20 \%)$ & $7644(22 \%)$ & $6890(34 \%)$ & $25,167(50 \%)$ \\
\hline
\end{tabular}

Year of Chemotherapy (Commercial), N (\%) 
Table 1 Characteristics of study population, by use of pegfilgrasim prophylaxis in cycle 1 (Continued)

\begin{tabular}{|c|c|c|c|c|}
\hline & \multicolumn{2}{|l|}{ Commercial } & \multicolumn{2}{|l|}{ Medicare } \\
\hline & No Pegfilgrastim Use & Pegfilgrastim Use & No Pegfilgrastim Use & Pegfilgrastim Use \\
\hline & $(n=16,512)$ & $(n=34,266)$ & $(n=20,195)$ & $(n=50,842)$ \\
\hline 2010-2011 & $7150(43 \%)$ & $13,308(39 \%)$ & - & - \\
\hline 2012-2013 & $6108(37 \%)$ & $13,108(38 \%)$ & - & - \\
\hline 2014-2015 & $3254(20 \%)$ & $7850(23 \%)$ & - & - \\
\hline \multicolumn{5}{|c|}{ Year of Chemotherapy (Medicare), N (\%) } \\
\hline 2007-2009 & - & - & $6268(31 \%)$ & $12,984(26 \%)$ \\
\hline 2010-2012 & - & - & 8615 (43\%) & $21,004(41 \%)$ \\
\hline 2013-2015 & - & - & $5312(26 \%)$ & $16,854(33 \%)$ \\
\hline
\end{tabular}

TAC docetaxel + doxorubicin + cyclophosphamide, $T C$ docetaxel + cyclophosphamide, $T C H$ docetaxel + cyclophosphamide + trastuzumab, $C H O P$ cyclophosphamide + doxorubicin + vincristine + prednisone with rituximab $(\mathrm{R})$

generally highest with TAC (OR $[\mathrm{vs}$ TC] $=2.7 ; 95 \% \mathrm{CI}$ 2.2-3.2) and $\mathrm{CHOP} \pm \mathrm{R}(\mathrm{OR}$ [vs TC] $=2.6$; 95\% CI 1.9$3.5)$, and increased with age (65-74 [vs 18-49]: OR = 1.3; 95\% CI $1.2-1.4 ; \geq 75$ [vs 18-49]: OR $=1.7$, 95\% CI $1.5-$ 2.0). Similar patterns were seen among Medicare patients: odds of FN across all cycles were substantially higher among those who had an FN event in a prior cycle (OR = 2.1 [95\% CI 2.1-2.2]), were highest with TAC (OR [vs $\mathrm{TC}]=2.1$ [95\% CI 1.9-2.3]) and $\mathrm{CHOP} \pm \mathrm{R}(\mathrm{OR}$ vs TC $=$ 1.6; $95 \%$ CI 1.6-1.7), and increased with age (75-84 [vs 65-74]: OR $=1.1$ [95\% CI 1.1-1.2]; $\geq 85$ [vs 65-74]: OR = 1.4 [95\% CI 1.3-1.4]). For both commercial and Medicare patients, results based on the narrow algorithm for FN suggest that elevated odds of FN among patients not receiving prophylaxis were even higher (Table 5).

\section{Discussion}

In this retrospective observational cohort study of patients with non-metastatic breast cancer or NHL who received chemotherapy with an intermediate to high risk for FN, we examined patterns of pegfilgrastim prophylaxis across cycles of chemotherapy, the influence of $\mathrm{FN}$ in one chemotherapy cycle on prophylaxis in subsequent cycles, and the incidence of FN among patients who did and did not receive prophylaxis. The findings from this examination-which were based on data from 50,778 commercial patients who received 190,622 cycles of chemotherapy and 71,037 Medicare patients who received 271,944 cyclessuggest that not only does a large minority of patients for whom prophylaxis is recommended fail to receive it (beginning in the first cycle), but that those patients have significantly higher odds of FN than patients who receive pegfilgrastim prophylaxis.

We found that approximately one in every three patients in this study did not receive pegfilgrastim prophylaxis in cycle 1-when FN risk is highest-and comparable proportions did not receive prophylaxis in subsequent cycles, thus exposing patients to a potentially fatal yet preventable complication of myelosuppressive chemotherapy [38]. We also found that while a history of FN does appear to increase the odds of receiving prophylactic coverage, more than one in seven commercial patients and one in four Medicare patients did not receive prophylaxis in a given cycle despite having $\mathrm{FN}$ in a prior cycle. Finally, we found that the adjusted incidence of FN in a given cycle was significantly higher among patients in our study population who did not receive prophylaxis in that cycle, highlighting the effectiveness of pegfilgrastim. We note that our findings are based on two large samples of patients with non-metastatic breast cancer and NHL who received chemotherapy regimens that are among the most commonly used in current clinical practice, and are largely consistent with the limited evidence that is currently available [39-47].

Two systematic reviews of randomized, controlled clinical trials comparing G-CSF prophylaxis with no prophylaxis showed significantly reduced risk of FN with pegfilgrastim $[39,43]$. Similar results have been observed in real-world data. In Hershman et al., which included 3123 randomly selected patients with solid tumors and lymphomas treated at 99 community practices in 2003, FN risk during the chemotherapy course was reported to be two-times higher among patients who did not receive primary prophylaxis (i.e., in cycle 1) versus those who did (adjusted OR $=2.0$ [95\% CI 1.4-2.9]) [46]. In a study of 239 women receiving adjuvant chemotherapy for early-stage breast cancer at a single clinic from 2009 to 2011, the FN odds ratio for patients not receiving GCSF primary prophylaxis (versus those receiving prophylaxis) was $2.6(p=0.002)$ [44]. The results of two other smaller studies were similar $[41,45]$. Accordingly, the results of these studies provide robust clinical and realworld evidence regarding the effectiveness of pegfilgrastim prophylaxis in the prevention of chemotherapy-induced FN among cancer patients of all ages.

Our results also are noteworthy given that clinical practice guidelines recommend administration of G-CSF in the 
Table 2 Use of pegfilgrastim prophylaxis in cycle 1, cycle 2, cycles 3+, last cycle, and all cycles, respectively, overall and by FN occurrence in a prior cycle*

\begin{tabular}{|c|c|c|c|c|c|c|c|c|}
\hline & \multicolumn{4}{|l|}{ Commercial } & \multicolumn{4}{|l|}{ Medicare } \\
\hline & \multirow[b]{2}{*}{$\begin{array}{l}\text { No. }(\%) \text { in Each } \\
\text { Category }\end{array}$} & \multicolumn{3}{|c|}{ Pegfilgrastim Use } & \multirow[b]{2}{*}{$\begin{array}{l}\text { No. (\%) in Each } \\
\text { Category }\end{array}$} & \multicolumn{3}{|c|}{ Pegfilgrastim Use } \\
\hline & & $\overline{\mathrm{No}}$ & Yes & $\begin{array}{l}\text { Unadjusted OR } \\
(95 \% \mathrm{Cl})\end{array}$ & & $\overline{\text { No }}$ & Yes & $\begin{array}{l}\text { Unadjusted OR } \\
(95 \% \mathrm{Cl})\end{array}$ \\
\hline \multicolumn{9}{|l|}{ Cycle 1} \\
\hline \multicolumn{9}{|c|}{ FN Events Prior to Cycle 1} \\
\hline No & $50,778(100 \%)$ & $\begin{array}{l}16,512 \\
(33 \%)\end{array}$ & $\begin{array}{l}34,266 \\
(67 \%)\end{array}$ & - & $71,037(100 \%)$ & $\begin{array}{l}20,195 \\
(28 \%)\end{array}$ & $\begin{array}{l}50,842 \\
(72 \%)\end{array}$ & - \\
\hline Yes & - & - & - & - & - & - & - & - \\
\hline Cycle 2 & 41,769 & $\begin{array}{l}11,719 \\
(28 \%)\end{array}$ & $\begin{array}{l}30,050 \\
(72 \%)\end{array}$ & & 59,412 & $\begin{array}{l}15,193 \\
(26 \%)\end{array}$ & $\begin{array}{l}44,219 \\
(74 \%)\end{array}$ & \\
\hline \multicolumn{9}{|c|}{ FN Events Prior to Cycle 2} \\
\hline No & $40,123(96 \%)$ & $\begin{array}{l}11,482 \\
(29 \%)\end{array}$ & $\begin{array}{l}28,641 \\
(71 \%)\end{array}$ & - & $55,488(93 \%)$ & $\begin{array}{l}14,483 \\
(26 \%)\end{array}$ & $\begin{array}{l}41,005 \\
(74 \%)\end{array}$ & - \\
\hline Yes & $1646(4 \%)$ & $237(14 \%)$ & $1409(86 \%)$ & $2.4(2.1-2.7)$ & $3924(7 \%)$ & $710(18 \%)$ & $3214(82 \%)$ & $1.6(1.5-1.7)$ \\
\hline $\begin{array}{l}\text { Cycles } \\
\geq 3\end{array}$ & 98,075 & $\begin{array}{l}24,834 \\
(25 \%)\end{array}$ & $\begin{array}{l}73,241 \\
(75 \%)\end{array}$ & & 141,495 & $\begin{array}{l}43,012 \\
(30 \%)\end{array}$ & $\begin{array}{l}98,483 \\
(70 \%)\end{array}$ & \\
\hline \multicolumn{9}{|c|}{ FN Events Prior to Cycle of Interest } \\
\hline No & $91,744(94 \%)$ & $\begin{array}{l}23,875 \\
(26 \%)\end{array}$ & $\begin{array}{l}67,869 \\
(74 \%)\end{array}$ & - & $126,424(89 \%)$ & $\begin{array}{l}39,360 \\
(31 \%)\end{array}$ & $\begin{array}{l}87,064 \\
(69 \%)\end{array}$ & - \\
\hline Yes & $6331(6 \%)$ & $959(15 \%)$ & $5372(85 \%)$ & $2.0(1.8-2.1)$ & $15,071(11 \%)$ & $\begin{array}{l}3652 \\
(24 \%)\end{array}$ & $\begin{array}{l}11,419 \\
(76 \%)\end{array}$ & $1.4(1.4-1.5)$ \\
\hline $\begin{array}{l}\text { Last } \\
\text { Cycle }\end{array}$ & 34,860 & $\begin{array}{l}10,188 \\
(29 \%)\end{array}$ & $\begin{array}{l}24,672 \\
(71 \%)\end{array}$ & & 51,989 & $\begin{array}{l}20,099 \\
(39 \%)\end{array}$ & $\begin{array}{l}31,890 \\
(61 \%)\end{array}$ & \\
\hline \multicolumn{9}{|c|}{ FN Events Prior to Last Cycle } \\
\hline No & $32,541(93 \%)$ & $\begin{array}{l}9774 \\
(30 \%)\end{array}$ & $\begin{array}{l}22,767 \\
(70 \%)\end{array}$ & - & $46,115(89 \%)$ & $\begin{array}{l}18,215 \\
(39 \%)\end{array}$ & $\begin{array}{l}27,900 \\
(61 \%)\end{array}$ & - \\
\hline Yes & $2319(7 \%)$ & $414(18 \%)$ & $1905(82 \%)$ & $2.0(1.8-2.2)$ & $5874(11 \%)$ & $\begin{array}{l}1884 \\
(32 \%)\end{array}$ & $3990(68 \%)$ & $1.4(1.3-1.5)$ \\
\hline $\begin{array}{l}\text { All } \\
\text { Cycles }\end{array}$ & 190,622 & $\begin{array}{l}53,065 \\
(28 \%)\end{array}$ & $\begin{array}{l}137,557 \\
(72 \%)\end{array}$ & & 271,944 & $\begin{array}{l}78,400 \\
(29 \%)\end{array}$ & $\begin{array}{l}193,544 \\
(71 \%)\end{array}$ & \\
\hline \multicolumn{9}{|c|}{ FN Events Prior to Cycle of Interest } \\
\hline No & $182,645(96 \%)$ & $\begin{array}{l}51,869 \\
(28 \%)\end{array}$ & $\begin{array}{l}130,776 \\
(72 \%)\end{array}$ & - & $252,949(93 \%)$ & $\begin{array}{l}74,038 \\
(29 \%)\end{array}$ & $\begin{array}{l}178,911 \\
(71 \%)\end{array}$ & - \\
\hline Yes & $7977(4 \%)$ & $\begin{array}{l}1196 \\
(15 \%)\end{array}$ & $6781(85 \%)$ & $2.2(2.1-2.4)$ & $18,995(7 \%)$ & $\begin{array}{l}4362 \\
(23 \%)\end{array}$ & $\begin{array}{l}14,633 \\
(77 \%)\end{array}$ & $1.4(1.3-1.4)$ \\
\hline
\end{tabular}

FN febrile neutropenia

*Only consecutive qualifying cycles, beginning with cycle 1, were considered in this analysis (e.g., in identifying FN events [broad definition] in a prior cycle of the course of interest)

first cycle when the risk of FN is $>20 \%$, and in subsequent cycles after FN or a dose-limiting neutropenic event where no prior G-CSF has been used. Unfortunately, the reasons why prophylaxis was not administered to so many patients in this study are unknown. While it is possible that other steps were taken to reduce the risk of FN, such as chemotherapy dose reductions (which are unobservable in the study database), it is also possible that patients were reluctant to return to the clinic to receive a pegfilgrastim injection on the day after chemotherapy $[48,49]$. Regardless of the reasons or the steps other than prophylaxis that were taken to prevent FN, our results demonstrate that patients with a history of FN were substantially more likely to experience a subsequent FN episode during their chemotherapy course, consistent with previous studies $[38,50]$.

There are several notable limitations to our study. A diagnosis code for FN does not exist, and thus FN was ascertained using operational algorithms and codes for neutropenia, fever, and infection that appeared during the pre-defined exposure period. We note that the recording of these diagnosis codes-especially those appearing earlier in the cycle (e.g., day 14 or earlier)-during the chemotherapy course increases the probability that the condition (i.e., neutropenia, 
Table 3 Crude incidence proportions for FN (broad definition) in cycle 1, cycle 2, cycles 3+, last cycle, and all cycles, respectively, by receipt of pegfilgrastim prophylaxis*

\begin{tabular}{|c|c|c|c|c|c|c|c|c|}
\hline & \multicolumn{4}{|l|}{ Commercial } & \multicolumn{4}{|l|}{ Medicare } \\
\hline & \multirow[b]{2}{*}{$\begin{array}{l}\text { No. (\%) in Each } \\
\text { Category }\end{array}$} & \multicolumn{3}{|l|}{ FN in Cycle } & \multirow[b]{2}{*}{$\begin{array}{l}\text { No. (\%) in Each } \\
\text { Category }\end{array}$} & \multicolumn{3}{|c|}{ FN in Cycle } \\
\hline & & No & Yes & $\begin{array}{l}\text { Unadjusted OR (95\% } \\
\text { Cl) }\end{array}$ & & No & Yes & $\begin{array}{l}\text { Unadjusted OR (95\% } \\
\text { Cl) }\end{array}$ \\
\hline Cycle 1 & 50,778 & $\begin{array}{l}48,157 \\
(95 \%)\end{array}$ & $\begin{array}{l}2621 \\
(5 \%)\end{array}$ & & 71,037 & $\begin{array}{l}64,249 \\
(90 \%)\end{array}$ & $\begin{array}{l}6788 \\
(10 \%)\end{array}$ & \\
\hline \multicolumn{9}{|c|}{ Use of Pegfilgrastim Prophylaxis } \\
\hline No & $16,512(33 \%)$ & $\begin{array}{l}15,234 \\
(92 \%)\end{array}$ & $\begin{array}{l}1278 \\
(8 \%)\end{array}$ & - & $20,195(28 \%)$ & $\begin{array}{l}17,901 \\
(89 \%)\end{array}$ & $\begin{array}{l}2294 \\
(11 \%)\end{array}$ & - \\
\hline Yes & $34,266(67 \%)$ & $\begin{array}{l}32,923 \\
(96 \%)\end{array}$ & $\begin{array}{l}1343 \\
(4 \%)\end{array}$ & $2.1(1.9-2.2)$ & $50,842(72 \%)$ & $\begin{array}{l}46,348 \\
(91 \%)\end{array}$ & $4494(9 \%)$ & $1.3(1.3-1.4)$ \\
\hline Cycle 2 & 41,769 & $\begin{array}{l}40,810 \\
(98 \%)\end{array}$ & $\begin{array}{l}959 \\
(2 \%)\end{array}$ & & 59,412 & $\begin{array}{l}56,343 \\
(95 \%)\end{array}$ & $3069(5 \%)$ & \\
\hline \multicolumn{9}{|c|}{ Use of Pegfilgrastim Prophylaxis } \\
\hline No & $11,719(28 \%)$ & $\begin{array}{l}11,353 \\
(97 \%)\end{array}$ & $\begin{array}{l}366 \\
(3 \%)\end{array}$ & - & $15,193(26 \%)$ & $\begin{array}{l}14,282 \\
(94 \%)\end{array}$ & $911(6 \%)$ & - \\
\hline Yes & $30,050(72 \%)$ & $\begin{array}{l}29,457 \\
(98 \%)\end{array}$ & $\begin{array}{l}593 \\
(2 \%)\end{array}$ & $1.6(1.4-1.8)$ & $44,219(74 \%)$ & $\begin{array}{l}42,061 \\
(95 \%)\end{array}$ & $2158(5 \%)$ & $1.2(1.1-1.3)$ \\
\hline $\begin{array}{l}\text { Cycles } \\
\geq 3\end{array}$ & 98,075 & $\begin{array}{l}96,006 \\
(98 \%)\end{array}$ & $\begin{array}{l}2069 \\
(2 \%)\end{array}$ & & 141,495 & $\begin{array}{l}134,363 \\
(95 \%)\end{array}$ & $7132(5 \%)$ & \\
\hline \multicolumn{9}{|c|}{ Use of Pegfilgrastim Prophylaxis } \\
\hline No & $24,834(25 \%)$ & $\begin{array}{l}24,229 \\
(98 \%)\end{array}$ & $\begin{array}{l}605 \\
(2 \%)\end{array}$ & - & $43,012(30 \%)$ & $\begin{array}{l}40,842 \\
(95 \%)\end{array}$ & $2170(5 \%)$ & - \\
\hline Yes & $73,241(75 \%)$ & $\begin{array}{l}71,777 \\
(98 \%)\end{array}$ & $\begin{array}{l}1464 \\
(2 \%)\end{array}$ & $1.2(1.1-1.3)$ & $98,483(70 \%)$ & $\begin{array}{l}93,521 \\
(95 \%)\end{array}$ & $4962(5 \%)$ & $1.0(1.0-1.1)$ \\
\hline $\begin{array}{l}\text { Last } \\
\text { Cycle }\end{array}$ & 34,860 & $\begin{array}{l}33,426 \\
(96 \%)\end{array}$ & $\begin{array}{l}1434 \\
(4 \%)\end{array}$ & & 51,989 & $\begin{array}{l}45,392 \\
(87 \%)\end{array}$ & $\begin{array}{l}6597 \\
(13 \%)\end{array}$ & \\
\hline \multicolumn{9}{|c|}{ Use of Pegfilgrastim Prophylaxis } \\
\hline No & $10,188(29 \%)$ & $9705(95 \%)$ & $\begin{array}{l}483 \\
(5 \%)\end{array}$ & - & $20,099(39 \%)$ & $\begin{array}{l}17,851 \\
(89 \%)\end{array}$ & $\begin{array}{l}2248 \\
(11 \%)\end{array}$ & - \\
\hline Yes & $24,672(71 \%)$ & $\begin{array}{l}23,721 \\
(96 \%)\end{array}$ & $\begin{array}{l}951 \\
(4 \%)\end{array}$ & $1.2(1.1-1.4)$ & $31,890(61 \%)$ & $\begin{array}{l}27,541 \\
(86 \%)\end{array}$ & $\begin{array}{l}4349 \\
(14 \%)\end{array}$ & $0.8(0.8-0.8)$ \\
\hline $\begin{array}{l}\text { All } \\
\text { Cycles }\end{array}$ & 190,622 & $\begin{array}{l}184,973 \\
(97 \%)\end{array}$ & $\begin{array}{l}5649 \\
(3 \%)\end{array}$ & & 271,944 & $\begin{array}{l}254,955 \\
(94 \%)\end{array}$ & $\begin{array}{l}16,989 \\
(6 \%)\end{array}$ & \\
\hline \multicolumn{9}{|c|}{ Use of Pegfilgrastim Prophylaxis } \\
\hline No & $53,065(28 \%)$ & $\begin{array}{l}50,816 \\
(96 \%)\end{array}$ & $\begin{array}{l}2249 \\
(4 \%)\end{array}$ & - & $78,400(29 \%)$ & $\begin{array}{l}73,025 \\
(93 \%)\end{array}$ & $5375(7 \%)$ & - \\
\hline Yes & 137,557 (72\%) & $\begin{array}{l}134,157 \\
(98 \%)\end{array}$ & $\begin{array}{l}3400 \\
(2 \%)\end{array}$ & $1.7(1.7-1.8)$ & $193,544(71 \%)$ & $\begin{array}{l}181,930 \\
(94 \%)\end{array}$ & $\begin{array}{l}11,614 \\
(6 \%)\end{array}$ & $1.2(1.1-1.2)$ \\
\hline
\end{tabular}

FN febrile neutropenia

*Only consecutive qualifying cycles, beginning with cycle 1, were considered in this analysis

infection, and/or fever) is associated with chemotherapy. In addition, ascertainment of FN in hospital was based on diagnosis codes alone as data on inpatient drug utilization are not available in the study databases. Because results from lab and other tests are unavailable in the study databases, and because other information (e.g., chemotherapy dose) is unavailable, not all FN risk factors were considered in analyses described herein. Because the validity of algorithms for identifying primary tumor type, metastatic disease, and comorbidity profiles has not been formally evaluated, their accuracy is unknown. Thus, to the extent that there may be unobserved systematic differences between patients who did (vs. did not) receive pegfilgrastim prophylaxis, study results may be confounded.

While approximately $20 \%$ of the commercial population and $40 \%$ of the Medicare population received an intermediate-risk chemotherapy regimen, it is likely that most of those patients would be classified as high-risk when considering patient risk factors (e.g., age $>65$ years, comorbidities) 
Table 4 Adjusted odds ratios for FN in cycle 1, cycle 2, cycles 3+, last cycle, and all cycles, respectively (Broad Definition of FN)

\begin{tabular}{|c|c|c|c|c|c|}
\hline & \multicolumn{5}{|c|}{ Adjusted Odds Ratio for FN (95\% Cl, p-value) $)^{*}$} \\
\hline & Cycle 1 & Cycle 2 & Cycles $\geq 3$ & Last Cycle & All Cycles \\
\hline \multicolumn{6}{|l|}{ Commercial } \\
\hline \multicolumn{6}{|l|}{ Independent Variables } \\
\hline No PEG Prophylaxis & $2.6(2.3-2.8,<0.001)$ & $1.9(1.6-2.2,<0.001)$ & $1.5(1.4-1.7,<0.001)$ & $1.5(1.4-1.7,<0.001)$ & $2.1(2.0-2.3,<0.001)$ \\
\hline FN in Prior Cycle & - & $3.2(2.6-3.9,<0.001)$ & $3.5(3.2-4.0,<0.001)$ & $2.4(2.0-2.8,<0.001)$ & $2.4(2.2-2.6,<0.001)$ \\
\hline \multicolumn{6}{|l|}{ Regimen } \\
\hline TAC & $2.9(2.6-3.3,<0.001)$ & $2.6(1.7-4.1,<0.001)$ & $1.7(1.4-1.9,<0.001)$ & $3.3(2.4-4.8,<0.001)$ & $2.7(2.2-3.2,<0.001)$ \\
\hline TC & - & - & - & - & - \\
\hline $\mathrm{TCH}$ & $1.1(1.0-1.2,0.229)$ & $2.2(1.4-3.3,<0.001)$ & $1.1(1.0-1.2,0.266)$ & $2.1(1.5-2.9,<0.001)$ & $1.4(1.2-1.6,<0.001)$ \\
\hline $\mathrm{CHOP} \pm \mathrm{R}$ & $1.3(1.2-1.5,<0.001)$ & $4.1(1.9-9.0,<0.001)$ & $1.7(1.5-1.9,<0.001)$ & $4.3(2.4-7.7,<0.001)$ & $2.6(1.9-3.5,<0.001)$ \\
\hline \multicolumn{6}{|l|}{ Age } \\
\hline $18-49$ & - & - & - & - & - \\
\hline $50-64$ & $1.0(0.9-1.1,0.377)$ & $1.0(0.9-1.2,0.884)$ & $1.1(1.0-1.2,0.242)$ & $1.3(1.1-1.4,0.002)$ & $1.0(1.0-1.1,0.409)$ \\
\hline $65-74$ & $1.3(1.2-1.5,<0.001)$ & $1.0(0.8-1.2,0.881)$ & $1.5(1.3-1.8,<0.001)$ & $1.7(1.4-2.1,<0.001)$ & $1.3(1.2-1.4,<0.001)$ \\
\hline$\geq 75$ & $1.7(1.4-2.1,<0.001)$ & $1.2(0.9-1.7,0.200)$ & $2.1(1.8-2.6,<0.001)$ & $3.4(2.7-4.3,<0.001)$ & $1.7(1.5-2.0,<0.001)$ \\
\hline \multicolumn{6}{|l|}{ Medicare } \\
\hline \multicolumn{6}{|l|}{ Independent Variables } \\
\hline No PEG Prophylaxis & $1.6(1.5-1.7,<0.001)$ & $1.6(1.5-1.8,<0.001)$ & $1.3(1.3-1.4,<0.001)$ & $1.0(0.9-1.0,0.507)$ & $1.5(1.4-1.5,<0.001)$ \\
\hline FN in Prior Cycle & - & $3.2(2.9-3.5,<0.001)$ & $2.9(2.7-3.1,<0.001)$ & $1.7(1.6-1.8,<0.001)$ & $2.1(2.1-2.2,<0.001)$ \\
\hline \multicolumn{6}{|l|}{ Regimen } \\
\hline TAC & $2.5(2.2-2.9,<0.001)$ & $1.9(1.5-2.4,<0.001)$ & $2.0(1.7-2.3,<0.001)$ & $2.2(1.9-2.6,<0.001)$ & $2.1(1.9-2.3,<0.001)$ \\
\hline TC & - & - & - & - & - \\
\hline $\mathrm{TCH}$ & $0.8(0.7-0.9,<0.001)$ & $0.9(0.8-1.0,0.127)$ & $0.8(0.7-0.9,<0.001)$ & $1.0(0.9-1.2,0.387)$ & $0.8(0.7-0.8,<0.001)$ \\
\hline $\mathrm{CHOP} \pm \mathrm{R}$ & $1.5(1.4-1.5,<0.001)$ & $1.8(1.7-2.0,<0.001)$ & $1.9(1.8-2.0,<0.001)$ & $1.8(1.7-2.0,<0.001)$ & $1.6(1.6-1.7,<0.001)$ \\
\hline \multicolumn{6}{|l|}{ Age } \\
\hline $65-74$ & - & - & - & - & - \\
\hline $75-84$ & $1.2(1.1-1.3,<0.001)$ & $1.0(0.9-1.1,0.709)$ & $1.1(1.1-1.2,<0.001)$ & $1.2(1.1-1.3,<0.001)$ & $1.1(1.1-1.2,<0.001)$ \\
\hline$\geq 85$ & $1.4(1.3-1.5,<0.001)$ & $1.2(1.1-1.4,<0.001)$ & $1.4(1.3-1.5,<0.001)$ & $1.8(1.6-1.9,<0.001)$ & $1.4(1.3-1.4,<0.001)$ \\
\hline
\end{tabular}

FN febrile neutropenia, PEG pegfilgrastim, TC docetaxel + cyclophosphamide, TAC docetaxel + doxorubicin + cyclophosphamide, TCH docetaxel + cyclophosphamide + trastuzumab, CHOP cyclophosphamide + doxorubicin + vincristine + prednisone, $R$, rituximab

*Adjusted for other characteristics of patients listed in Table 1; additional covariates selected via backward selection method

and thus recommended to receive G-CSF prophylaxis [51]. It is possible, however, that a small percentage of patients in the study population may have had a projected risk of FN $<20 \%$. While over $95 \%$ of CSF prophylaxis patients received pegfilgrastim, a small percentage received one of the daily agents (principally filgrastim) and thus overall CSF use is somewhat higher than reported estimates. While the study period overlapped for a few months with the availability of the Neulasta ${ }^{\circ}$ Onpro $^{\circ}$ kit-an on-body injector that is applied 1 day and delivers pegfilgrastim approximately $27 \mathrm{~h}$ later-these patients were excluded from analyses if their pegfilgrastim administration was identified as having occurred on the same day as chemotherapy. Based on the timing of the data relative to Onpro ${ }^{\circ}$ availability, the net impact of such exclusions was likely small. Because our study population was limited to patients with non-metastatic breast cancer or NHL who received selected intermediate/high-risk chemotherapy regimens, study results may not be generalizable to other cancers or other regimens.

\section{Conclusions}

In this retrospective evaluation of non-metastatic breast cancer and NHL patients receiving select chemotherapy regimens with an intermediate/highrisk for FN, a sizeable portion of patients did not receive G-CSF prophylaxis, and an important minority did not receive G-CSF prophylaxis in cycles following FN. Patients not receiving G-CSF prophylaxis had a 
Table 5 Adjusted odds ratios for FN in cycle 1, cycle 2, cycles 3+, last cycle, and all cycles, respectively (Narrow Definition of FN)

\begin{tabular}{|c|c|c|c|c|c|}
\hline & \multicolumn{5}{|c|}{ Adjusted Odds Ratio for FN (95\% Cl, p-value) } \\
\hline & Cycle 1 & Cycle 2 & Cycles $\geq 3$ & Last Cycle & All Cycles \\
\hline \multicolumn{6}{|l|}{ Commercial } \\
\hline \multicolumn{6}{|l|}{ Independent Variables } \\
\hline No PEG Prophylaxis & $4.2(3.8-4.7,<0.001)$ & $4.1(3.3-4.9,<0.001)$ & $3.3(2.9-3.8,<0.001)$ & $3.2(2.7-3.8,<0.001)$ & $4.2(3.9-4.5,<0.001)$ \\
\hline FN in Prior Cycle & - & $5.1(3.7-7.0,<0.001)$ & $5.9(4.9-7.0,<0.001)$ & $3.9(3.0-5.0,<0.001)$ & $3.1(2.6-3.5,<0.001)$ \\
\hline \multicolumn{6}{|l|}{ Regimen } \\
\hline TAC & $4.4(3.8-5.1,<0.001)$ & $2.2(1.6-3.2,<0.001)$ & $3.0(2.4-3.8,<0.001)$ & $2.7(2.0-3.6,<0.001)$ & $4.1(3.3-5.2,<0.001)$ \\
\hline TC & - & - & - & - & - \\
\hline $\mathrm{TCH}$ & $1.0(0.9-1.1,0.963)$ & $1.4(1.1-1.8,0.009)$ & $1.2(1.0-1.4,0.130)$ & $1.2(0.9-1.5,0.191)$ & $1.3(1.0-1.6,0.040)$ \\
\hline $\mathrm{CHOP} \pm \mathrm{R}$ & $1.4(1.2-1.6,<0.001)$ & $2.0(1.5-2.7,<0.001)$ & $3.5(2.9-4.4,<0.001)$ & $1.7(1.4-2.1,<0.001)$ & $3.0(2.0-4.5,<0.001)$ \\
\hline \multicolumn{6}{|l|}{ Age } \\
\hline $18-49$ & - & - & - & - & - \\
\hline $50-64$ & $1.1(1.0-1.2,0.216)$ & $1.1(0.9-1.4,0.332)$ & $1.0(0.9-1.2,0.671)$ & $1.3(1.1-1.6,0.010)$ & $1.1(1.0-1.2,0.207)$ \\
\hline $65-74$ & $1.5(1.3-1.8,<0.001)$ & $1.2(0.8-1.6,0.354)$ & $1.4(1.1-1.8,0.002)$ & $1.6(1.2-2.1,0.003)$ & $1.4(1.3-1.6,<0.001)$ \\
\hline$\geq 75$ & $2.1(1.7-2.7,<0.001)$ & $1.7(1.1-2.6,0.012)$ & $2.0(1.5-2.6,<0.001)$ & $4.0(2.9-5.5,<0.001)$ & $2.1(1.7-2.4,<0.001)$ \\
\hline \multicolumn{6}{|l|}{ Medicare } \\
\hline \multicolumn{6}{|l|}{ Independent Variables } \\
\hline No PEG Prophylaxis & $2.0(1.9-2.2,<0.001)$ & $2.2(2.0-2.5,<0.001)$ & $1.6(1.5-1.8,<0.001)$ & $1.3(1.2-1.4,<0.001)$ & $1.9(1.8-2.0,<0.001)$ \\
\hline FN in Prior Cycle & - & $4.6(4.0-5.3,<0.001)$ & $4.1(3.8-4.4,<0.001)$ & $2.4(2.1-2.6,<0.001)$ & $2.7(2.5-2.9,<0.001)$ \\
\hline \multicolumn{6}{|l|}{ Regimen } \\
\hline TAC & $3.1(2.7-3.7,<0.001)$ & $2.5(1.9-3.5,<0.001)$ & $2.8(2.3-3.5,<0.001)$ & $2.5(2.0-3.1,<0.001)$ & $2.7(2.4-3.0,<0.001)$ \\
\hline TC & - & - & - & - & - \\
\hline $\mathrm{TCH}$ & $0.6(0.6-0.7,<0.001)$ & $0.7(0.5-0.9,0.001)$ & $0.6(0.5-0.8,<0.001)$ & $0.7(0.6-0.8,<0.001)$ & $0.6(0.5-0.6,<0.001)$ \\
\hline $\mathrm{CHOP} \pm \mathrm{R}$ & $1.6(1.5-1.7,<0.001)$ & $2.1(1.9-2.5,<0.001)$ & $2.9(2.6-3.2,<0.001)$ & $2.1(1.9-2.3,<0.001)$ & $1.9(1.8-2.0,<0.001)$ \\
\hline \multicolumn{6}{|l|}{ Age } \\
\hline $65-74$ & - & - & - & - & - \\
\hline $75-84$ & $1.2(1.1-1.3,<0.001)$ & $1.0(0.8-1.1,0.759)$ & $1.1(1.0-1.2,0.011)$ & $1.2(1.1-1.4,<0.001)$ & $1.1(1.1-1.2,<0.001)$ \\
\hline$\geq 85$ & $1.4(1.3-1.6,<0.001)$ & $1.1(1.0-1.3,0.047)$ & $1.2(1.1-1.4,<0.001)$ & $1.6(1.5-1.8,<0.001)$ & $1.3(1.3-1.4,<0.001)$ \\
\hline
\end{tabular}

FN febrile neutropenia, PEG pegfilgrastim, TC docetaxel + cyclophosphamide, TAC docetaxel + doxorubicin + cyclophosphamide, TCH docetaxel + cyclophosphamide + trastuzumab, $C H O P$ cyclophosphamide + doxorubicin + vincristine + prednisone, $\mathrm{R}$ rituximab

${ }^{*}$ Adjusted for other characteristics of patients listed in Table 1; additional covariates selected via backward selection method

markedly higher incidence of FN versus those who received G-CSF prophylaxis. Accordingly, appropriate use of G-CSF prophylaxis may reduce exposure to a potentially fatal but largely preventable complication of myelosuppressive chemotherapy.

\section{Additional file}

Additional file 1: Online supplement:study methods. (DOCX $143 \mathrm{~kb}$ )

\section{Abbreviations}

ANC: Absolute neutrophil count; $\mathrm{CHOP} \pm \mathrm{R}$ : Cyclophosphamide + doxorubicin + vincristine + prednisone \pm rituximab; CMS: Centers for Medicare and Medicaid Services; CSF: Colony-stimulating factor; FN: Febrile neutropenia; GCSF: Granulocyte colony-stimulating factor; GEE: Generalized estimating equations; HIPAA: Health Insurance Portability and Accountability Act; IRB: Institutional Review Board; NHL: Non-Hodgkin's lymphoma; OR: Odds ratio; PAl: Policy Analysis Inc:; RIFs: Research Identifiable Files; TAC: Docetaxel + doxorubicin + cyclophosphamide; TC: Docetaxel + cyclophosphamide; $\mathrm{TCH}$ : Docetaxel + carboplatin + trastuzumab

\section{Acknowledgements}

We acknowledge Micah Robinson, PhD, and Kerri Hebard-Massey, PhD of Amgen Inc. for providing medical writing support.

\section{Authors' contributions}

The contribution of each of these persons to this study is as follows: [1] conception and design (DW, DC, MB), acquisition of data (DW, DC, MB), analysis or interpretation of data (DW, $R D, A H, C B, R B, D C, A L, M B, G H L)$; and [2] preparation of manuscript (DW, RD, $A H)$, critical review of manuscript (CB, $\mathrm{RB}, \mathrm{DC}, \mathrm{AL}, \mathrm{MB}, \mathrm{GHL})$. All authors have read and approved the final version of the manuscript.

\section{Authors' information}

Not applicable. 


\section{Funding}

Funding for this research was provided by Amgen Inc. to Policy Analysis Inc. (PAI). The sponsor reviewed and approved the study protocol and the final version of the manuscript. Data management, processing, and analyses were conducted by PAI. All final analytic decisions were made by the authors, and the final version of the manuscript was approved by all authors.

\section{Availability of data and materials}

The data are proprietary, provided by third-party vendors, and the authors do not have permission to disseminate these data without approval of the vendors.

\section{Ethics approval and consent to participate}

The data extracts were de-identified prior to their release to study investigators, and thus their use for health services research is compliant with the HIPAA Privacy Rule and federal guidance on Public Welfare and the Protection of Human Subjects and is exempt from IRB review.

\section{Consent for publication}

Not applicable.

\section{Competing interests}

$D W, R D, A H$, and AL are employed by Policy Analysis Inc. (PAI). CB, RB, DC, and $\mathrm{MB}$ are employed by, and own stock in, Amgen Inc. GHL is the principal investigator on a research grant to Fred Hutchinson Cancer Research Center from Amgen Inc.

\section{Author details}

'Policy Analysis Inc. (PAI), Four Davis Court, Brookline, MA 02445, USA. ${ }^{2}$ Amgen Inc., Thousand Oaks, CA, USA. ${ }^{3}$ Fred Hutchinson Cancer Research Center and the University of Washington, Seattle, WA, USA.

\section{Received: 18 February 2019 Accepted: 2 August 2019} Published online: 09 August 2019

\section{References}

1. Bonadonna G, Moliterni A, Zambetti M, Daidone MG, Pilotti S, Gianni L, et al. 30 years' follow up of randomised studies of adjuvant CMF in operable breast cancer: cohort study. BMJ. 2005;330(7485):217.

2. Bosly A, Bron D, Van Hoof A, De Bock R, Berneman Z, Ferrant A, et al. Achievement of optimal average relative dose intensity and correlation with survival in diffuse large B-cell lymphoma patients treated with CHOP. Ann Hematol. 2008;87(4):277-83.

3. Caggiano V, Weiss RV, Rickert TS, Linde-Zwirble WT. Incidence, cost, and mortality of neutropenia hospitalization associated with chemotherapy. Cancer. 2005;103(9):1916-24.

4. Kuderer NM, Dale DC, Crawford J, Cosler LE, Lyman GH. Mortality, morbidity, and cost associated with febrile neutropenia in adult cancer patients. Cancer. 2006;106(10):2258-66.

5. Kwak LW, Halpern J, Olshen RA, Horning SJ. Prognostic significance of actual dose intensity in diffuse large-cell lymphoma: results of a treestructured survival analysis. J Clin Oncol. 1990;8(6):963-77.

6. Lyman GH. Impact of chemotherapy dose intensity on cancer patient outcomes. J Natl Compr Cancer Netw. 2009;7(1):99-108.

7. Lyman GH, Dale DC, Crawford J. Incidence and predictors of low doseintensity in adjuvant breast cancer chemotherapy: a nationwide study of community practices. J Clin Oncol. 2003;21 (24):4524-31.

8. Lyman GH, Dale DC, Tomita D, Whittaker S, Crawford J. A retrospective evaluation of chemotherapy dose intensity and supportive care for earlystage breast cancer in a curative setting. Breast Cancer Res Treat. 2013; 139(3):863-72.

9. Lyman GH, Michels SL, Reynolds MW, Barron R, Tomic KS, Yu J. Risk of mortality in patients with cancer who experience febrile neutropenia. Cancer. 2010;116(23):5555-63.

10. Shayne M, Crawford J, Dale DC, Culakova E, Lyman GH, Group ANCS. Predictors of reduced dose intensity in patients with early-stage breast cancer receiving adjuvant chemotherapy. Breast Cancer Res Treat. 2006; 100(3):255-62.

11. Smith TJ, Khatcheressian J, Lyman GH, Ozer H, Armitage JO, Balducci L, et al. 2006 update of recommendations for the use of white blood cell growth factors: an evidence-based clinical practice guideline. J Clin Oncol. 2006 24(19):3187-205.

12. Tan H, Tomic K, Hurley D, Daniel G, Barron R, Malin J. Comparative effectiveness of colony-stimulating factors for febrile neutropenia: a retrospective study. Curr Med Res Opin. 2011;27(1):79-86 Epub 2010/11/26.

13. Klastersky J, De Naurois J, Rolston K, Rapoport B, Maschmeyer G, Aapro M, et al. Management of febrile neutropaenia: ESMO clinical practice guidelines. Ann Oncol. 2016;27(suppl_5):v111-8.

14. Aapro MS, Bohlius J, Cameron DA, Dal Lago L, Donnelly JP, Kearney N, et al. 2010 update of EORTC guidelines for the use of granulocyte-colony stimulating factor to reduce the incidence of chemotherapy-induced febrile neutropenia in adult patients with lymphoproliferative disorders and solid tumours. Eur J Cancer. 2011;47(1):8-32.

15. Weycker D, Malin J, Barron R, Edelsberg J, Kartashov A, Oster G. Comparative effectiveness of filgrastim, pegfilgrastim, and sargramostim as prophylaxis against hospitalization for neutropenic complications in patients with cancer receiving chemotherapy. Am J Clin Oncol. 2012;35(3):267-74 Epub 2011/03/08.

16. Weycker D, Li X, Figueredo J, Barron R, Tzivelekis S, Hagiwara M. Risk of chemotherapy-induced febrile neutropenia in cancer patients receiving pegfilgrastim prophylaxis: does timing of administration matter? Support Care Cancer. 2016;24(5):2309-16 Epub 2015/11/27.

17. Weycker D, Li X, Barron R, Li Y, Reiner M, Kartashov A, et al. Risk of chemotherapy-induced febrile neutropenia with early discontinuation of pegfilgrastim prophylaxis in US clinical practice. Support Care Cancer. 2016; 24(6):2481-90 Epub 2015/12/17.

18. Potosky AL, Malin JL, Kim B, Chrischilles EA, Makgoeng SB, Howlader N, et al. Use of colony-stimulating factors with chemotherapy: opportunities for cost savings and improved outcomes. J Natl Cancer Inst. 2011;103(12):979-82 Epub 2011/06/15.

19. Weycker D, Malin J, Kim J, Barron R, Edelsberg J, Kartashov A, et al. Risk of hospitalization for neutropenic complications of chemotherapy in patients with primary solid tumors receiving pegfilgrastim or filgrastim prophylaxis: a retrospective cohort study. Clin Ther. 2009;31(5):1069-81 Epub 2009/06/23.

20. Morrison VA, Wong M, Hershman D, Campos LT, Ding B, Malin J. Observational study of the prevalence of febrile neutropenia in patients who received filgrastim or pegfilgrastim associated with 3-4 week chemotherapy regimens in community oncology practices. J Manag Care Pharm. 2007;13(4):337-48 Epub 2007/05/18.

21. Weycker D, Hackett J, Edelsberg JS, Oster G, Glass AG. Are shorter courses of filgrastim prophylaxis associated with increased risk of hospitalization? Ann Pharmacother. 2006;40(3):402-7 Epub 2006/02/24.

22. Scott SD, Chrischilles EA, Link BK, Delgado DJ, Fridman M, Stolshek BS. Days of prophylactic filgrastim use to reduce febrile neutropenia in patients with non-Hodgkin's lymphoma treated with chemotherapy. J Manag Care Pharm. 2003;9(2 Suppl):15-21 Epub 2003/11/14.

23. Weycker D, Bensink M, Lonshteyn A, Doroff R, Chandler D. Risk of chemotherapy-induced febrile neutropenia by day of pegfilgrastim prophylaxis in US clinical practice from 2010 to 2015. Curr Med Res Opin. 2017:33(12):2107-13 Epub 2017/09/30.

24. Weycker D, Li X, Tzivelekis S, Atwood M, Garcia J, Li Y, et al. Burden of chemotherapy-induced febrile neutropenia hospitalizations in US clinical practice, by use and patterns of prophylaxis with colony-stimulating factor. Support Care Cancer. 2017;25(2):439-47 Epub 2016/10/14.

25. Schnipper LE, Smith TJ, Raghavan D, Blayney DW, Ganz PA, Mulvey TM, et al. American Society of Clinical Oncology identifies five key opportunities to improve care and reduce costs: the top five list for oncology. I Clin Oncol. 2012:30(14):1715-24 Epub 2012/04/12.

26. Waters GE, Corrigan P, Gatesman M, Smith TJ. Comparison of pegfilgrastim prescribing practice to national guidelines at a university hospital outpatient oncology clinic. J Oncol Pract. 2013;9(4):203-6 Epub 2013/08/15.

27. Kuderer NM, Lyman GH. Personalized medicine and cancer supportive care: appropriate use of colony-stimulating factor support of chemotherapy. J Natl Cancer Inst. 2011;103(12):910-3 Epub 2011/06/15.

28. Weycker D, Bensink M, Lonshteyn A, Doroff R, Chandler D. Use of colonystimulating factor primary prophylaxis and incidence of febrile neutropenia from 2010 to 2016: a longitudinal assessment. Curr Med Res Opin. 2019; 35(6):1073-80.

29. Naeim A, Henk HJ, Becker $L$, et al. Pegfilgrastim prophylaxis is associated with a lower risk of hospitalization of cancer patients than filgrastim prophylaxis: a retrospective United States claims analysis of granulocyte colony-stimulating factors (G-CSF). BMC Cancer. 2013;13:11. 
30. Siena S, Piccart MJ, Holmes FA, et al. A combined analysis of two pivotal randomized trials of a single dose of pegfilgrastim per chemotherapy cycle and daily Filgrastim in patients with stage II-IV breast cancer. Oncol Rep. 2003;10(3):715-24.

31. Pinto L, Liu Z, Doan $Q$, et al. Comparison of pegfilgrastim with filgrastim on febrile neutropenia, grade IV neutropenia and bone pain: a meta-analysis of randomized controlled trials. Curr Med Res Opin. 2007;23(9):2283-95.

32. Cooper KL, Madan J, Whyte S, Stevenson MD, Akehurst RL. Granulocyte colony-stimulating factors for febrile neutropenia prophylaxis following chemotherapy: systematic review and metaanalysis. BMC Cancer. 2011;11:404.

33. Wang L, Baser O, Kutikova L, Page JH, Barron R. The impact of primary prophylaxis with granulocyte colony-stimulating factors on febrile neutropenia during chemotherapy: a systematic review and meta-analysis of randomized controlled trials. Support Care Cancer. 2015:23(11):3131-40.

34. Lambertini M, Ferreira AR, Del Mastro L, Danesi R, Pronzato P. Pegfilgrastim for the prevention of chemotherapy-induced febrile neutropenia in patients with solid tumors. Expert Opin Biol Ther. 2015;15(12):1799-817.

35. Weycker D, Bensink M, Wu H, Doroff R, Chandler D. Risk of chemotherapyinduced febrile neutropenia with early discontinuation of pegfilgrastim prophylaxis based on real-world data from 2010 to 2015. Curr Med Res Opin. 2017;33(12):2115-20.

36. US Department of Health and Human Services (2018) Code of Federal Regulations: Title 45, public welfare; Part 46, protection of human subjects. http://www.hhs.gov/ohrp/humansubjects/guidance/45cfr46.html. Accessed 4 July 2019

37. Weycker D, Sofrygin O, Seefeld K, Deeter RG, Legg J, Edelsberg J. Technical evaluation of methods for identifying chemotherapy-induced febrile neutropenia in healthcare claims databases. BMC Health Serv Res. 2013;13:60

38. Culakova E, Thota R, Poniewierski MS, Kuderer NM, Wogu AF, Dale DC, et al. Patterns of chemotherapy-associated toxicity and supportive care in US oncology practice: a nationwide prospective cohort study. Cancer med. 2014;3(2):434-44 Epub 2014/04/08

39. Kuderer NM, Dale DC, Crawford J, Lyman GH. Impact of primary prophylaxis with granulocyte colony-stimulating factor on febrile neutropenia and mortality in adult cancer patients receiving chemotherapy: a systematic review. J Clin Oncol. 2007;25(21):3158-67 Epub 2007/07/20.

40. Aarts MJ, Peters FP, Mandigers CM, Dercksen MW, Stouthard JM, Nortier HJ, et al. Primary granulocyte colony-stimulating factor prophylaxis during the first two cycles only or throughout all chemotherapy cycles in patients with breast cancer at risk for febrile neutropenia. J Clin Oncol. 2013;31(34):4290-6 Epub 2013/05/01.

41. Lee S, Knox A, Zeng IS, Coomarasamy C, Blacklock H, Issa S. Primary prophylaxis with granulocyte colony-stimulating factor (GCSF) reduces the incidence of febrile neutropenia in patients with non-Hodgkin lymphoma $(\mathrm{NHL})$ receiving $\mathrm{CHOP}$ chemotherapy treatment without adversely affecting their quality of life: cost-benefit and quality of life analysis. Support Care Cancer. 2013;21(3):841-6 Epub 2012/09/14.

42. Langeberg WJ, Siozon CC, Page JH, Morrow PK, Chia VM. Use of pegfilgrastim primary prophylaxis and risk of infection, by chemotherapy cycle and regimen, among patients with breast cancer or non-Hodgkin's lymphoma. Support Care Cancer. 2014;22(8):2167-75 Epub 2014/03/22.

43. Wang L, Baser O, Kutikova L, Page JH, Barron R. The impact of primary prophylaxis with granulocyte colony-stimulating factors on febrile neutropenia during chemotherapy: a systematic review and meta-analysis of randomized controlled trials. Support Care Cancer. 2015;23(11):3131-40 Epub 2015/03/31.

44. Altwairgi AK, Hopman WM, Mates M. Real-world impact of granulocyte-colony stimulating factor on febrile neutropenia. Curr Oncol. 2013;20(3):e171-9.

45. Bradley AM, Deal AM, Buie LW, van Deventer H. Neutropenia-associated outcomes in adults with acute myeloid leukemia receiving cytarabine consolidation chemotherapy with or without granulocyte colonystimulating factor. Pharmacother. 2012;32(12):1070-7.

46. Hershman D, Hurley D, Wong M, Morrison VA, Malin JL. Impact of primary prophylaxis on febrile neutropenia within community practices in the US. J Med Econ. 2009;12(3):203-10.

47. National Comprehensive Cancer Network. NCCN Clinical Practice Guidelines in Oncology (NCCN Guidelines ${ }^{\circledast}$ ). Myeloid Growth Factors. Version 1. 2018. https://www.ncen.org/professionals/physician_gls/pdf/growthfactors.pdf. cited 25 July 2018.
48. Stephens JM, Li X, Reiner M, Tzivelekis S. Annual patient and caregiver burden of oncology clinic visits for granulocyte-colony stimulating factor therapy in the US. J Med Econ. 2016;19(5):537-47 Epub 2016/01/09.

49. Stephens JM, Bensink M, Bowers C, Hollenbeak CS. Travel burden associated with granulocyte colony-stimulating factor administration in a Medicare aged population: a geospatial analysis. Curr Med Res Opin. 2018;34(8):135160 Epub 2017/07/20

50. Lyman GH, Dale DC, Culakova E, Poniewierski MS, Wolff DA, Kuderer NM, et al. The impact of the granulocyte colony-stimulating factor on chemotherapy dose intensity and cancer survival: a systematic review and meta-analysis of randomized controlled trials. Ann Oncol. 2013;24(10):247584 Epub 2013/06/22.

51. Weycker D, Li X, Barron R, Wu H, Morrow PK, Xu H, et al. Importance of risk factors for febrile neutropenia among patients receiving chemotherapy regimens not classified as high-risk in guidelines for myeloid growth factor use. J Natl Compr Cancer Netw. 2015;13(8):979-86.

\section{Publisher's Note}

Springer Nature remains neutral with regard to jurisdictional claims in published maps and institutional affiliations.
Ready to submit your research? Choose BMC and benefit from:

- fast, convenient online submission

- thorough peer review by experienced researchers in your field

- rapid publication on acceptance

- support for research data, including large and complex data types

- gold Open Access which fosters wider collaboration and increased citations

- maximum visibility for your research: over 100M website views per year

At $\mathrm{BMC}$, research is always in progress.

Learn more biomedcentral.com/submissions 